\title{
Statistical properties of the repeating fast radio burst source FRB 121102
}

\author{
Bing $\mathrm{Li}^{1-3 *}$, Long-Biao $\mathrm{Li}^{4}$, Zhi-Bin Zhang ${ }^{5}$, Jin-Jun Geng, ${ }^{1,6}, \mathrm{Li}-$ Ming Song ${ }^{2,3}$, Yong-Feng Huang ${ }^{1,6 *}$ and Yuan-Pei Yang \\ ${ }^{1}$ School of Astronomy and Space Science, Nanjing University, Nanjing 210023, China \\ ${ }^{2}$ Laboratory for Particle Astrophysics, Institute of High Energy Physics, Beijing 100049, China \\ ${ }^{3}$ Key Laboratory of Particle Astrophysics, Chinese Academy of Sciences, Beijing 100049, China \\ ${ }^{4}$ School of Mathematics and Physics, Hebei University of Engineering, Handan 056005, China \\ ${ }^{5}$ College of Physics and Engineering, Qufu Normal University, Qufu 273165, China \\ ${ }^{6}$ Key Laboratory of Modern Astronomy and Astrophysics (Nanjing University), Ministry of Education, China \\ ${ }^{7}$ Kavli Institute for Astronomy and Astrophysics, Peking University, Beijing 100871, China \\ ${ }^{8}$ National Astronomical Observatories, Chinese Academy of Sciences, Beijing 100012, China
}

\section{Article Info}

\section{*Corresponding authors: \\ Bing Li}

School of Astronomy and Space Science

Nanjing University

Nanjing 210023, China

E-mail: libing@ihep.ac.cn

\section{Yong-Feng Huang}

Professor

Key Laboratory of Modern Astronomy and Astrophysics

School of Astronomy and Space Science Nanjing University

Ministry of Education, China

E-mail: hyf@nju.edu.cn

Received: December 20, 2018

Accepted: December 31, 2018

Published: January 23, 2019

Citation: Li B, Li LB, Zhang ZB, et al. Statistical properties of the repeating fast radio burst source FRB 121102. Int J Cosmol Astron Astrophys. 2019; 1(1): 22-32. doi: 10.18689/ijcaa-1000108

\section{Copyright: (c) 2019 The Author(s). This work is licensed under a Creative Commons Attribution 4.0 International License, which permits unrestricted use, distribution, and reproduction in any medium, provided the original work is properly cited.}

Published by Madridge Publishers

\begin{abstract}
Currently, FRB 121102 is the only fast radio burst source that was observed to give out bursts repeatedly. It shows a high repeating rate, with more than one hundred bursts being spotted, but with no obvious periodicity in the activities. Thanks to its repetition, the source was well localized with a subarcsecond accuracy, leading to a red shift measurement of about 0.2. FRB 121102 is a unique source that can help us understand the enigmatic nature of fast radio bursts. In this study, we analyze the characteristics of the waiting times between bursts from FRB 121102. It is found that there is a clear bimodal distribution for the waiting times. While most waiting times cluster at several hundred seconds, a small portion of the waiting times are strikingly in the range of 2-40 millisecond. More interestingly, it is found that the waiting time does not correlate with the burst intensity, either for the preceding burst or for the subsequent burst. It strongly indicates that the repeating bursts should be generated by some external mechanisms, but not internal mechanisms. As a result, the models involving collisions between small bodies and neutron stars could be competitive mechanisms for this interesting source.
\end{abstract}

Keywords: Fast radio bursts (FRBs); FRB 121102; Galactic pulsars; Neutron Stars; Black Holes

\section{Introduction}

Fast radio bursts (FRBs) are intense extragalactic radio bursts with a flux of Jy level and with short durations of about several millisecond. Their origin and trigger mechanism is still an open question. The first FRB event (010724) was once named according to the discoverer as "Lorimer Burst" [1]. After about one decade, more than 60 FRBs have been found by terrestrial radio telescopes [2]'. FRBs have anomalously high dispersion measures (DMs), significantly exceeding the expected Milky Way contribution along the line of sight, which is contrary to Galactic pulsars [3-10] (but note that low DM has also been hinted from two FRBs [11, 12] and a candidate (FRB 141113) [13]). It indicates that FRBs are of extragalactic or even cosmological origin, rather than of Galactic origin [1418]. Their bright millisecond radio pulses with a high scattering effect point to extremely high brightness temperatures, which implies that luminous coherent emission processes around compact objects should be involved, which might include the curvature radiation (also known as the "antenna" mechanism) [19-21], or the synchrotron maser emission [22-25]. However, the exact mechanism is still enigmatic and greatly debated [26-27]. 
Numerous theoretical models have been proposed to account for such an enigmatic class of radio transients. The number of models is almost comparable to that of the observed FRBs up to date [28, 29]. Most of those progenitor models involve compact objects (e.g., Neutron Stars (NS), Black Holes (BH) and White Dwarfs (WD)) outside of the Milky Way. Popular mechanisms include: mergers of binary NS [3032], or binary WD [33], or NS-WD binary [34,35], or NS/WD$\mathrm{BH}$ binary [36-38], the interactions of pulsar- $\mathrm{BH}$ systems [39] or Kerr-Newman BH-BH [40,41], collapse of compact objects (e.g., collapse of NS [42-46], collapse of strange star crust [47], giant pulses/flares from magnetars or young pulsars [48-51], and other supernovae interrelated theories [22, 52-55]. A few other models include: mechanisms related to active galactic nuclei(AGN) and Kerr-BH or Strange Star interactions [56-60], collisions between NSs and small bodies [61-64], Axion star collides with a NS/BH [65-67], interactions between Axions and compact bodies [68-70], explosions such as star quakes [71], primordial BHs/Planck stars collapse to form white holes $[72,73]$, lightning/wandering in pulsars $[16,74]$, or NS combing $[75,76]$, and so on. In short, while the true mechanisms of FRBs are still unclear, it is possible that multiple populations of FRBs might exist [77-79]. For a summary of FRB progenitor models, see the recent article of Platts et al. [80] and the online version of a tabulated summary ${ }^{2}$.

'Petroffet al.2016 catalogued and updated all published sample of FRBs, see their online catalog-http://www.frbcat. org

\section{${ }^{2}$ http://frbtheorycat.org}

In general, the above models could be grouped into two different categories [81, 82], i.e. catastrophic models and noncatastrophic models. Following this idea, a key issue is then whether the observed FRBs are repetitive or not. Strangely enough, among the more than 60 FRB sources, only one event, i.e. FRB 121102 is observed to repeatedly burst out, which actually has produced hundreds of bursts so far. For all the other FRB sources, no indication of repetition was observed although long-term monitoring had been extensively carried out [1, 3, 9, 11, 83-86]. However, strictly speaking, it still remains obscure how many FRBs are repetitive and whether their repetition is similar to the unique FRB 121102 or not. In this study, we will mainly concentrate on the repeating event of FRB 121102.

FRB 121102 was initially discovered through the 305-m Arecibo telescope Pulsar Survey Project (ALFA) [87]. It is the first and the only known burst that has been successfully identified to be associated with a host galaxy, a small lowmetallicity star-forming dwarf galaxy at $z=0.19273 \pm 0.00008$ [89-92]. It is also the only burst with a repeating behavior [92]. Hundreds of additional bursts have been observed from the same direction toward FRB 121102 (the position is known to sub-arc second precision) and the measured DMs of all these bursts (between $553-569 \mathrm{pc} \mathrm{cm}^{-3}$ ) are consistent with the first burst of 121102. These consequent observations were made discontinuously by many terrestrial radio telescopes such as the Arecibo telescope (AO), the Green Bank Hydrogen
telescope(GBT), the Karl G. Jansky Very Large Array(VLA), the 100-m Effelsberg telescope (Eff), and the Apertif Radio Transient System (ARTS), at multiple radio bands [77, 88, 92101]. They all reveal no additional waveband sporadic emission [88, 93, 102-103], and there is no evidence for any periodicity [100]. It is interesting that a cosmological origin is confirmed for FRB 121102 confirms with the red shift measurement. The catastrophic models are also ruled out for this source. FRB 121102 is associated with a variable radio source with a continuum non-thermal spectrum. It seems to be a low-luminosity AGN or other kind of unknown peculiar source [88]. An AGN scenario thus remains possible [56-59], but it could also be due to other mechanisms such as a young neutron star with pulsar wind nebula [48-51], or a neutron star interacting with small bodies [62-64].

For a sporadically repeating astrophysical phenomenon, the temporal information or quantities (e.g., duration, elapsed time, periodicity, episodic time) of outbursts play an important role in understanding the central engine, and revealing the radiation mechanism and energy dissipation processes. For instance, the previous researches on the non-stationary Poisson process of soft gamma-ray repeaters [104-106], pulsar glitches [107-109], anomalous X-ray pulsars (AXPs) $[110,111], X$-ray flares in $\gamma$ ray burst afterglows $[112,113]$, can give us helpful references. A time-variable system always shows some kind of irregularity. Especially the time interval between two adjacent bursts, which is known as the waiting time (here after WT or $\Delta \mathrm{t}$ ), is an important parameter that could provide valuable information on the central engine.

Previously, Gu et al.(2016) established a relation between the waiting time and the mass transfer rate of accretion in a NS-WD binary merger [34], which can be some what related to the observed behaviors of FRB 121102. Katz (2018) found the distribution of the waiting times between bursts of FRB 121102 are apparently far from a Poissonian form, and proposed that such a distribution may be consistent with a precessing jet launched from a NS or a $\mathrm{BH}$ accretion disc [114]. Wang. et al.(2018) found that the energy and waiting time distributions of FRB121102 derived from the paper [99] show an earthquake-like behavior. They argued that those bursts could be powered by some star quake-like mechanisms [71]. Palaniswamy et al.(2018) compared 40 bursts of FRB 121102 with other non-repeating FRBs on the observed waiting time-flux ratio plane. They found that their distribution is well separated from other FRBs in the plane, suggesting that there could be multiple populations of FRBs [78]. In this study, with more observed bursts (on the level of hundred) from the repeating source FRB 121102, we re-analyze the waiting time statistics. Especially, we study the correlation between the waiting time and the flux and other parameters of the repeating bursts. It is expected that these new studies will help us understand the nature of this unique source.

The structure of our paper is organized as follows. In Section II, we collect the detailed observational data for the repeated bursts from FRB 121102. We then calculate the waiting times for the available events. In Section III, we derive 
the waiting time distribution and analysis its correlation with other parameters. The implication of our study for the nature of the repeater is presented. Finally, Section IV presents our conclusions and some further discussion.

\section{The Observations and Samples of FRB 121102}

FRB 121102 is currently the only source to exhibit repeating bursts among the FRB population. From the literature, we have collected all the observational data of the observed bursts from this source. An overview of the available observations is listed in (Table 1). In this table, we present the date of observations, the telescope names, the starting time of the observation, the duration of the corresponding monitoring, number of bursts observed during the observational campaign, the number of available waiting times during the period, and references. Note that in each observational campaign, the source usually could be monitored continuously for several thousands (or up to several hours in several cases). A meaningful waiting time could be derived only for two bursts that are observed in the same observational campaign. For example, if 5 bursts were observed in a continuous observational campaign, then 4 waiting time could be derived from these 5 bursts.

Up to now, there are thirty-five continuous observation campaigns on FRB 121102 that resulted in successful detection of at least one fast radio burst from the source.A lot of bursts are detected in Observation Campaigns 33 and 34, i.e. 46 and 47 bursts were observed, respectively. In summary, more than one burst was detected in 17 observations (see the sixth column in Table 1), providing us with 171 bursts in total, as listed in table 2 at the end of this article.

Table 1. Log of the observations on FRB 121102

\begin{tabular}{|l|l|l|l|l|l|l|l|}
\hline Obs. & \multicolumn{1}{|c|}{ Date } & Telescope & Start Time & $\begin{array}{c}\text { Obs. } \\
\text { Length }\end{array}$ & $\begin{array}{c}\text { Num- } \\
\text { ber }\end{array}$ & $\begin{array}{c}\text { Waiting } \\
\text { time }\end{array}$ & Reference \\
\hline $\begin{array}{c}\text { num- } \\
\text { ber }\end{array}$ & $\begin{array}{c}\text { yyy/mm/ } \\
\text { dd }\end{array}$ & /Receiver & hh/mm/ss & $t_{\text {obs }}$ & $\begin{array}{c}\text { of } \\
\text { Bursts }\end{array}$ & number & \\
\hline 1 & $2012 / 11 / 02$ & AO/ALFA & $06: 38: 13$ & $\sim 200$ & 1 & 0 & {$[88,93,94]$} \\
\hline 2 & $2015 / 05 / 17$ & AO/ALFA & $17: 45: 38$ & 1002 & 2 & 1 & {$[93]$} \\
\hline 3 & $2015 / 06 / 02$ & AO/ALFA & $16: 38: 47$ & 1002 & 2 & 1 & {$[93]$} \\
\hline 4 & $2015 / 06 / 02$ & AO/ALFA & $17: 48: 52$ & 1002 & 6 & 5 & {$[93]$} \\
\hline 5 & $2015 / 11 / 13$ & GBT/S-band & $07: 42: 09$ & 3000 & 1 & 0 & {$[94]$} \\
\hline 6 & $2015 / 11 / 19$ & GBT/S-band & $10: 14: 57$ & 3000 & 4 & 3 & {$[94]$} \\
\hline 7 & $2015 / 12 / 08$ & AO/L-wide & $04: 43: 24$ & 3625 & 1 & 0 & {$[94]$} \\
\hline 8 & $2016 / 08 / 20$ & Eff/5 GHz & $09: 23: 40$ & 1200 & 3 & 2 & {$[101]$} \\
\hline 9 & $2016 / 08 / 23$ & VLA/3 GHz & $17: 26: 28$ & 3240 & 1 & 0 & {$[89,96]$} \\
\hline 10 & $2016 / 09 / 02$ & VLA/3 GHz & $15: 52: 17$ & 3240 & 2 & 1 & {$[89,96]$} \\
\hline 11 & $2016 / 09 / 07$ & VLA/3 GHz & $10: 14: 50$ & 7200 & 1 & 0 & {$[89,96]$} \\
\hline 12 & $2016 / 09 / 12$ & VLA/3 GHz & $09: 15: 19$ & 7200 & 1 & 0 & {$[89,96]$} \\
\hline $13^{\text {a }}$ & $2016 / 09 / 14$ & VLA/3 GHz & $09: 20: 23$ & 7200 & 1 & 0 & {$[89,96]$} \\
\hline 14 & $2016 / 09 / 15$ & VLA/3 GHz & $09: 16: 29$ & 7200 & 1 & 0 & {$[89,96]$} \\
\hline 15 & $2016 / 09 / 16$ & GBT/S-band & $03: 59: 12$ & 14452 & 2 & 1 & {$[98]$} \\
\hline $16^{b}$ & $2016 / 09 / 17$ & VLA/3 GHz & $08: 59: 20$ & 7200 & 1 & 0 & {$[89,96]$} \\
\hline 17 & $2016 / 09 / 18$ & GBT/S-band & $04: 02: 15$ & 14269 & 2 & 1 & {$[98]$} \\
\hline $18^{\text {a }}$ & $2016 / 09 / 18$ & VLA/3 GHz & $08: 59: 27$ & 7200 & 1 & 0 & {$[89,96]$} \\
\hline 19 & $2016 / 12 / 25$ & AO4.1-4.9 GHz & $02: 48: 51.998$ & 6703 & 10 & 9 & {$[78]$} \\
\hline
\end{tabular}

\begin{tabular}{|l|l|l|l|r|c|c|l|}
\hline 20 & $2016 / 12 / 26$ & AO4.1-4.9 GHz & $02: 44: 21.998$ & 6806 & 5 & 4 & {$[78]$} \\
\hline $21 c$ & $2017 / 01 / 11$ & GBT/S-band & $23: 13: 56$ & 19869 & 6 & 5 & {$[98]$} \\
\hline $22^{c}$ & $2017 / 01 / 12$ & AO/L-wide & $01: 46: 27$ & 6270 & 4 & 3 & {$[98]$} \\
\hline 23 & $2017 / 01 / 16$ & Eff/L-band & $16: 14: 20$ & 14400 & 3 & 2 & {$[95]$} \\
\hline 24 & $2017 / 01 / 19$ & AO4.1-4.9GHz & $01: 25: 03$ & 5901 & 1 & 0 & {$[78]$} \\
\hline 25 & $2017 / 01 / 19$ & Eff/L-band & $16: 05: 40$ & 12630 & 5 & 4 & {$[95]$} \\
\hline 26 & $2017 / 01 / 25$ & Eff/L-band & $16: 22: 00$ & 12600 & 4 & 3 & {$[95]$} \\
\hline 27 & $2017 / 02 / 15$ & AO/WE.G-1.38 & & & 1 & 0 & {$[99]$} \\
\hline 28 & $2017 / 02 / 19$ & Eff/L-band & $14: 28: 40$ & 10800 & 1 & 0 & {$[95]$} \\
\hline 19 & $2017 / 02 / 22$ & AO/WE.G-1.38 & & & 1 & 0 & {$[99]$} \\
\hline 30 & $2017 / 02 / 22$ & $\begin{array}{l}\text { GO/ZE.G-1.38 } \\
\text { GHz }\end{array}$ & & & 1 & 0 & {$[99]$} \\
\hline 31 & $2017 / 02 / 24$ & $\begin{array}{l}\text { AO/WE.G-1.38 } \\
\text { GHz }\end{array}$ & & & 1 & 0 & {$[99]$} \\
\hline 32 & $2017 / 03 / 02$ & $\begin{array}{l}\text { AONWE.G-1.38 } \\
\text { GHz }\end{array}$ & & & 1 & 0 & {$[99]$} \\
\hline 33 & $2017 / 08 / 26$ & GBT/C-band & $13: 51: 44$ & 1800 & $46^{\mathrm{d}}$ & 45 & {$[100,102]$} \\
\hline 34 & $2017 / 08 / 26$ & GBT/C-band & $14: 21: 59$ & 1800 & $47^{\mathrm{d}}$ & 46 & {$[100,102]$} \\
\hline 35 & $2017 / 08 / 31$ & ARTS/L-wide & $06: 23: 37$ & 3600 & 1 & 0 & {$[97]$} \\
\hline
\end{tabular}

Note: Each observation campaign is marked with a sequence number according to the start time, as shown in Column 1. Column 2 is the observation date; Column 3 presents the receiver and telescope name; Column 4 gives the start time of the observation; Column 5 gives observation duration; Column 6 gives the number of FRBs observed during the campaign, and Column 7 is the number of available waiting times; Column 8 provides the references.

aln these observations, the source was monitored at AO/Lwide but the observations gave non-detection results.

${ }^{b}$ In these observations, one burst was detected both by the VLA fast-dump observations at $3 \mathrm{GHz}$ band and Arecibo observations with $\mathrm{L}$-wide receiver at a frequency range of 1.15 $-1.73 \mathrm{GHz}$.

In these observations, the observation time was crossing, and two bursts were detected both by GBT/S-band and AO/Lband receiver [97].

dObservations by the GBT/4 - $8 \mathrm{GHz}$ (or C-band) receiver with Breakthrough Listen digital backend. Due to successful application of deep learning [101], the number of bursts is tremendous above $5.2 \mathrm{GHz}$. Note that in these two observations, 21 bursts have been published earlier by Gajjar et al. 2018 [99]. Here we only consider Zhang's results [101].

We have calculated the waiting times for those successive bursts detected in a continuous observation. The waiting time (WT, $\Delta t$ ) is defined as the time interval between two neighboring and non-piled-up bursts in a continuous and uninterrupted observation campaign. For the repetitive fast radio bursts from FRB 121102, the observed profiles are composed of only a single pulse. In our calculations, we ignored the time dilation factor of $1+z$, which is not large and will not affect our analysis. As a result, the number of waiting times available is 136, as listed in Column 4 of (Table 2). In table 2, we also listed some other important parameters such as the peak flux density, the fluence, the duration, etc. 
International Journal of Cosmology, Astronomy and Astrophysics

Table 2. The waiting time and a few key parameters of the repeating bursts from FRB 121102.

For the references of the observational data, please see (Table 1).

\begin{tabular}{|c|c|c|c|c|c|c|c|c|c|c|c|}
\hline Burst & BPT & TOA & WT & Center frequency & Bandwidth & $S$ & $\sigma S$ & $F$ & $\sigma_{F}$ & $w$ & $\sigma w$ \\
\hline No. & (MJD) & (day) & (s) & $(\mathrm{MHz})$ & $(\mathrm{MHz})$ & (Jy) & & (Jyms) & & (ms) & \\
\hline 1 & 56233.282837008 & & & 1375 & 322 & 0.040 & 0.008 & 0.10 & 0.012 & 3.3 & 0.30 \\
\hline 2 & 57159.737600835 & 926.4548 & 572.2085 & 1375 & 322 & 0.030 & 0.006 & 0.10 & 0.012 & 3.8 & 0.40 \\
\hline 3 & 57159.744223619 & 926.4614 & & 1375 & 322 & 0.030 & 0.006 & 0.10 & 0.012 & 3.3 & 0.40 \\
\hline 4 & 57175.693143232 & 942.4103 & 568.9089 & 1375 & 322 & 0.040 & 0.008 & 0.20 & 0.012 & 4.6 & 0.30 \\
\hline 5 & 57175.699727826 & 942.4169 & & 1375 & 322 & 0.020 & 0.004 & 0.09 & 0.030 & 8.7 & 1.50 \\
\hline 6 & 57175.742576706 & 942.4597 & 22.6919 & 1375 & 322 & 0.020 & 0.004 & 0.06 & 0.008 & 2.8 & 0.40 \\
\hline 7 & 57175.742839344 & 942.4600 & 57.9782 & 1375 & 322 & 0.020 & 0.004 & 0.06 & 0.028 & 6.1 & 1.40 \\
\hline 8 & 57175.743510388 & 942.4607 & 186.2304 & 1375 & 322 & 0.140 & 0.028 & 0.90 & 0.014 & 6.6 & 0.10 \\
\hline 9 & 57175.745665832 & 942.4628 & 169.2592 & 1375 & 322 & 0.050 & 0.01 & 0.30 & 0.015 & 6.0 & 0.30 \\
\hline 10 & 57175.747624851 & 942.4648 & 57.2326 & 1375 & 322 & 0.050 & 0.01 & 0.20 & 0.025 & 8.0 & 0.50 \\
\hline 11 & 57175.748287265 & 942.4655 & & 1375 & 322 & 0.310 & 0.062 & 1.00 & 0.012 & 3.06 & 0.04 \\
\hline 12 & 57339.356046005567 & 1106.0732 & & 2000 & 800 & 0.040 & & 0.20 & 0.045 & 6.73 & 1.12 \\
\hline 13 & 57345.447691250090 & 1112.1649 & 414.4327 & 2000 & 800 & 0.060 & & 0.40 & 0.034 & 6.10 & 0.57 \\
\hline 14 & 57345.452487925162 & 1112.1697 & 441.2775 & 2000 & 800 & 0.040 & & 0.20 & 0.040 & 6.14 & 1.00 \\
\hline 15 & 57345.457595303807 & 1112.1748 & 416.2582 & 2000 & 800 & 0.020 & & 0.08 & 0.028 & 4.30 & 1.40 \\
\hline 16 & 57345.462413106565 & 1112.1796 & & 2000 & 800 & 0.090 & & 0.60 & 0.032 & 5.97 & 0.35 \\
\hline 17 & 57364.204632665605 & 1130.9218 & & 1430 & 700 & 0.030 & 0.006 & 0.09 & 0.007 & 2.50 & 0.23 \\
\hline 18 & 57620.392218422 & 1387.1094 & 160.3673 & 4850 & & 0.3 & 0.04 & 0.15 & 0.05 & 0.5 & 0.10 \\
\hline 19 & 57620.394074525 & 1387.1112 & 480.0102 & 4850 & & 0.2 & 0.04 & 0.12 & 0.044 & 0.6 & 0.10 \\
\hline 20 & 57620.399630199 & 1387.1168 & & 4850 & & 0.1 & 0.02 & 0.17 & 0.064 & 1.7 & 0.30 \\
\hline 21 & 57623.74402686 & 1390.4612 & & 3000 & 512 & 0.120 & & 0.60 & & 5.0 & \\
\hline 22 & 57633.67986367 & 1400.3970 & 1321.5493 & 3000 & 500 & 0.670 & & 3.35 & & 5.0 & \\
\hline 23 & 57633.69515938 & 1400.4123 & & 3000 & 500 & 0.025 & & 0.13 & & 5.0 & \\
\hline 24 & 57638.49937435 & 1405.2165 & & 3000 & 500 & 0.063 & & 0.32 & & 5.0 & \\
\hline 25 & 57643.45730263 & 1410.1745 & & 3000 & 500 & 0.326 & & 1.63 & & 5.0 & \\
\hline 26 & 57645.42958602 & 1412.1467 & & 3000 & 500 & 0.039 & & 0.20 & & 5.0 & \\
\hline 27 & 57646.46600650 & 1413.1832 & & 3000 & 500 & 0.050 & & 0.25 & & 5.0 & \\
\hline 28 & 57647.232346450619 & 1413.9495 & 0.0374 & 2000 & 800 & 0.360 & & 0.32 & 0.022 & 2.16 & 0.06 \\
\hline 29 & 57647.232346883015 & 1413.9495 & & 2000 & 800 & 0.080 & & 0.16 & 0.020 & 1.94 & 0.25 \\
\hline 30 & 57648.43691490 & 1415.1541 & & 3000 & 500 & 0.086 & 0.014 & 0.43 & & 5.0 & \\
\hline 31 & 57649.173812898174 & 1415.8910 & 3836.1884 & 2000 & 800 & 0.360 & & 1.32 & 0.025 & 3.45 & 0.07 \\
\hline 32 & 57649.218213226581 & 1415.9354 & & 2000 & 800 & 0.290 & & 0.34 & 0.020 & 0.88 & 0.07 \\
\hline 33 & 57649.45175697 & 1416.1689 & & 3000 & 500 & 0.159 & 0.006 & 0.80 & & 5.0 & \\
\hline 34 & 57747.1295649013 & 1513.8467 & 658.5214 & 4500 & 800 & 0.9 & 0.18 & 0.7 & 0.14 & 0.8 & 0.01 \\
\hline 35 & 57747.1371866766 & 1513.8543 & 784.8879 & 4500 & 800 & 0.3 & 0.06 & 0.2 & 0.04 & 0.85 & 0.01 \\
\hline 36 & 57747.1462710273 & 1513.8634 & 458.1716 & 4500 & 800 & 0.8 & 0.16 & 0.2 & 0.04 & 0.22 & 0.01 \\
\hline 37 & 57747.1515739398 & 1513.8687 & 250.0029 & 4500 & 800 & 0.2 & 0.04 & 0.09 & 0.018 & 0.55 & 0.01 \\
\hline 38 & 57747.1544674918 & 1513.8716 & 503.0038 & 4500 & 800 & 0.2 & 0.04 & 0.1 & 0.02 & 0.76 & 0.01 \\
\hline 39 & 57747.1602892954 & 1513.8775 & 4.7001 & 4500 & 800 & 1.8 & 0.36 & 0.05 & 0.01 & 0.03 & 0.01 \\
\hline 40 & 57747.1603436945 & 1513.8775 & 473.8184 & 4500 & 800 & 0.6 & 0.12 & 0.2 & 0.04 & 0.31 & 0.01 \\
\hline 41 & 57747.1658277033 & 1513.8830 & 47.2859 & 4500 & 800 & 0.4 & 0.08 & 0.5 & 0.1 & 1.36 & 0.01 \\
\hline 42 & 57747.1663749941 & 1513.8835 & 828.7868 & 4500 & 800 & 0.2 & 0.04 & 0.3 & 0.06 & 1.92 & 0.01 \\
\hline 43 & 57747.1759674338 & 1513.8931 & & 4500 & 800 & 0.2 & 0.04 & 0.2 & 0.04 & 0.98 & 0.01 \\
\hline 44 & 57748.1256436428 & 1514.8428 & 2408.9006 & 4500 & 800 & 0.1 & 0.02 & 0.1 & 0.02 & 0.95 & 0.01 \\
\hline 45 & 57748.1535244366 & 1514.8707 & 146.0587 & 4500 & 800 & 0.4 & 0.08 & 0.2 & 0.04 & 0.42 & 0.01 \\
\hline 46 & 57748.1552149312 & 1514.8724 & 206.7319 & 4500 & 800 & 0.8 & 0.16 & 0.6 & 0.12 & 0.78 & 0.01 \\
\hline 47 & 57748.1576076618 & 1514.8748 & 1562.9040 & 4500 & 800 & 1.2 & 0.24 & 0.2 & 0.04 & 0.15 & 0.01 \\
\hline 48 & 57748.1756968287 & 1514.8929 & & 4500 & 800 & 0.4 & 0.08 & 0.4 & 0.08 & 0.54 & 0.01 \\
\hline 49 & 57765.049526345771 & 1531.7667 & 1319.0573 & 2000 & 800 & 0.17 & & 0.33 & 0.015 & 1.40 & 0.09 \\
\hline 50 & 57765.064793212950 & 1531.7820 & 367.5706 & 2000 & 800 & 0.38 & & 0.83 & 0.015 & 1.79 & 0.04 \\
\hline 51 & 57765.069047502300 & 1531.7862 & 2745.8220 & 2000 & 800 & 0.20 & & 0.62 & 0.024 & 2.97 & 0.12 \\
\hline 52 & 57765.100827849608 & 1531.8180 & 0.0008 & 1380 & 800 & 0.09 & 0.018 & 0.37 & 0.010 & 4.29 & 0.11 \\
\hline 53 & 57765.100827859293 & 1531.8180 & 678.4977 & 2000 & 800 & 0.09 & & 0.18 & 0.025 & 2.46 & 0.28 \\
\hline 54 & 57765.108680842022 & 1531.8258 & 1045.2119 & 1380 & 800 & 0.02 & 0.004 & 0.03 & 0.002 & 3.69 & 0.11 \\
\hline 55 & 57765.120778202479 & 1531.8379 & 0.0002 & 1380 & 800 & 0.02 & 0.004 & 0.05 & 0.009 & 4.34 & 0.44 \\
\hline 56 & 57765.120778204779 & 1531.8379 & 1358.2429 & 2000 & 800 & 0.56 & & 1.08 & 0.017 & 1.36 & 0.03 \\
\hline 57 & 57765.136498608757 & 1531.8537 & 590.8832 & 2000 & 800 & 0.11 & & 0.22 & 0.019 & 1.68 & 0.17 \\
\hline 58 & 57765.143337535257 & 1531.8605 & & 1380 & 800 & 0.03 & 0.006 & 0.10 & 0.010 & 3.66 & 0.32 \\
\hline
\end{tabular}


International Journal of Cosmology, Astronomy and Astrophysics

\begin{tabular}{|c|c|c|c|c|c|c|c|c|c|c|c|}
\hline 59 & 57769.6881141561 & 1536.4053 & 1225.7660 & 1360 & 300 & 0.7 & 0.1 & 2.6 & 0.40 & 3.56 & 0.09 \\
\hline 60 & 57769.702301263 & 1536.4195 & 5328.0127 & 1360 & 300 & 0.17 & 0.03 & 0.6 & 0.10 & 3.400 & 0.40 \\
\hline 61 & 57769.7639680761 & 1536.4811 & & 1360 & 300 & 0.8 & 0.1 & 1.7 & 0.30 & 2.050 & 0.06 \\
\hline 62 & 57772.1290302972 & 1538.8462 & & 4500 & 800 & 0.8 & 0.16 & 0.6 & 0.12 & 0.74 & 0.01 \\
\hline 63 & 57772.6649755688 & 1539.3821 & 1990.5243 & 1360 & 300 & 0.36 & 0.05 & 0.7 & 0.10 & 2.100 & 0.10 \\
\hline 64 & 57772.688014045 & 1539.4052 & 6089.6034 & 1360 & 300 & 0.11 & 0.02 & 0.35 & 0.09 & 3.200 & 0.70 \\
\hline 65 & 57772.758495566 & 1539.4757 & 337.0257 & 1360 & 300 & 0.25 & 0.04 & 1.3 & 0.20 & 5.100 & 0.30 \\
\hline 66 & 57772.762396326 & 1539.4796 & 1928.7907 & 1360 & 300 & 0.22 & 0.03 & 0.4 & 0.07 & 1.800 & 0.20 \\
\hline 67 & 57772.784720292 & 1539.5019 & & 1360 & 300 & 0.21 & 0.03 & 0.4 & 0.09 & 1.900 & 0.30 \\
\hline 68 & 57778.6885027615 & 1545.4057 & 5855.1556 & 1360 & 300 & 0.39 & 0.06 & 1 & 0.20 & 2.600 & 0.10 \\
\hline 69 & 57778.756270766 & 1545.4734 & 0.0341 & 1360 & 300 & 0.11 & 0.02 & 0.23 & 0.06 & 2.100 & 0.40 \\
\hline 70 & 57778.756271161 & 1545.4734 & 3708.5134 & 1360 & 300 & 0.18 & 0.03 & 0.5 & 0.10 & 2.900 & 0.30 \\
\hline 71 & 57778.799193770 & 1545.5164 & & 1360 & 300 & 0.21 & 0.03 & 0.38 & 0.07 & 1.800 & 0.20 \\
\hline 72 & 57799.983175660 & 1566.7003 & & 1380 & 800 & 1.4 & 0.28 & 8.022 & 1.604 & 5.73 & \\
\hline 73 & 57803.692917989 & 1570.4101 & & 1360 & 300 & 0.21 & 0.03 & 0.7 & 0.10 & 3.500 & 0.30 \\
\hline 74 & 57806.964250780 & 1573.6814 & 1769.3225 & 1380 & 800 & 1.6 & 0.32 & 3.936 & 0.787 & 2.46 & \\
\hline 75 & 57806.984729050 & 1573.7019 & & 1380 & 800 & 1.5 & 0.3 & 5.535 & 1.107 & 3.69 & \\
\hline 76 & 57808.002785850 & 1574.7199 & & 1380 & 800 & 0.79 & 0.158 & 2.915 & 0.583 & 3.69 & \\
\hline 77 & 57814.946985200 & 1581.6641 & & 1380 & 800 & 0.47 & 0.094 & 0.541 & 0.108 & 1.15 & \\
\hline 78 & 57991.4099040442 & 1758.1276 & 2.2185 & 7020 & 3900 & 0.7635 & 0.0105 & 0.6061 & 0.0212 & 1.43 & \\
\hline 79 & 57991.4099297208 & 1758.1271 & 7.9579 & 7050 & 2300 & 0.0348 & 0.0081 & 0.0256 & 0.0201 & 2.15 & \\
\hline 80 & 57991.4100218264 & 1758.1272 & 4.1287 & 7020 & 2400 & 0.0539 & 0.0083 & 0.0505 & 0.0168 & 1.43 & \\
\hline 81 & 57991.4100696130 & 1758.1272 & 128.6319 & 6170 & 2500 & 0.0541 & 0.0084 & 0.0906 & 0.0225 & 2.51 & \\
\hline 82 & 57991.4115584078 & 1758.1287 & 45.5501 & 5560 & 2500 & 0.0457 & 0.0084 & 0.053 & 0.019 & 1.79 & \\
\hline 83 & 57991.4120856083 & 1758.1292 & 19.5705 & 5470 & 1400 & 0.0318 & 0.0064 & 0.0435 & 0.0144 & 1.79 & \\
\hline 84 & 57991.4123121182 & 1758.1295 & 39.1048 & 7020 & 2400 & 0.0449 & 0.0083 & 0.0264 & 0.0146 & 1.08 & \\
\hline 85 & 57991.4127647200 & 1758.1299 & 0.0143 & 5550 & 3900 & 0.0972 & 0.0105 & 0.0664 & 0.0212 & 1.43 & \\
\hline 86 & 57991.4127648858 & 1758.1299 & 13.9614 & 5450 & 2900 & 0.0569 & 0.0091 & 0.0673 & 0.0206 & 1.79 & \\
\hline 87 & 57991.4129264759 & 1758.1301 & 8.0693 & 5440 & 2000 & 0.0719 & 0.0092 & 0.1461 & 0.0294 & 3.58 & \\
\hline 88 & 57991.4130198711 & 1758.1302 & 29.6054 & 7030 & 1800 & 0.138 & 0.0072 & 0.0566 & 0.0103 & 0.72 & \\
\hline 89 & 57991.4133625264 & 1758.1305 & 8.3149 & 7030 & 1800 & 0.0448 & 0.0072 & 0.0468 & 0.0146 & 1.43 & \\
\hline 90 & 57991.4134587642 & 1758.1306 & 21.4177 & 7060 & 4000 & 0.4521 & 0.0106 & 0.3632 & 0.0186 & 1.08 & \\
\hline 91 & 57991.4137066537 & 1758.1309 & 11.2669 & 7110 & 4000 & 0.3421 & 0.0106 & 0.2628 & 0.0185 & 1.08 & \\
\hline 92 & 57991.4138370580 & 1758.1310 & 198.3099 & 7030 & 4000 & 0.3182 & 0.0106 & 0.2646 & 0.0215 & 1.43 & \\
\hline 93 & 57991.4161323109 & 1758.1332 & 4.5723 & 7010 & 2900 & 0.0328 & 0.0091 & 0.0353 & 0.0207 & 1.79 & \\
\hline 94 & 57991.4161852305 & 1758.1333 & 1.9554 & 7020 & 2400 & 0.0375 & 0.0083 & 0.0462 & 0.0188 & 1.79 & \\
\hline 95 & 57991.4162078619 & 1758.1334 & 19.7797 & 5720 & 2000 & 0.0384 & 0.0075 & 0.0229 & 0.0132 & 1.08 & \\
\hline 96 & 57991.4164367935 & 1758.1336 & 16.9835 & 5470 & 3900 & 0.0689 & 0.0106 & 0.0362 & 0.0185 & 1.08 & \\
\hline 97 & 57991.4166333622 & 1758.1338 & 54.9513 & 7020 & 3900 & 0.5637 & 0.0105 & 0.3492 & 0.0184 & 1.08 & \\
\hline 98 & 57991.4172693722 & 1758.1344 & 9.6220 & 5800 & 2500 & 0.0732 & 0.0084 & 0.0526 & 0.0148 & 1.08 & \\
\hline 99 & 57991.4173807378 & 1758.1345 & 28.8562 & 6450 & 1900 & 0.0723 & 0.0074 & 0.0295 & 0.0106 & 0.72 & \\
\hline 100 & 57991.4177147221 & 1758.1349 & 13.0318 & 5750 & 2500 & 0.0542 & 0.0084 & 0.0352 & 0.0147 & 1.08 & \\
\hline 101 & 57991.4178655533 & 1758.1350 & 65.8063 & 6410 & 3900 & 0.136 & 0.0105 & 0.0811 & 0.0184 & 1.08 & \\
\hline 102 & 57991.4186272003 & 1758.1358 & 34.6894 & 7610 & 3900 & 0.1288 & 0.0105 & 0.0978 & 0.0213 & 1.43 & \\
\hline 103 & 57991.4190286984 & 1758.1362 & 36.3906 & 5750 & 2000 & 0.0541 & 0.0075 & 0.0401 & 0.0151 & 1.43 & \\
\hline 104 & 57991.4194498856 & 1758.1366 & 0.0052 & 5750 & 1700 & 0.0508 & 0.0069 & 0.0446 & 0.0139 & 1.43 & \\
\hline 105 & 57991.4194499461 & 1758.1366 & 0.0019 & 7110 & 1000 & 0.04 & 0.0054 & 0.0213 & 0.0095 & 1.08 & \\
\hline 106 & 57991.4194499686 & 1758.1366 & 1.0948 & 6220 & 2600 & 0.0496 & 0.0068 & 0.0362 & 0.012 & 1.08 & \\
\hline 107 & 57991.4194626399 & 1758.1366 & 0.0193 & 5720 & 2000 & 0.039 & 0.0075 & 0.0256 & 0.017 & 1.79 & \\
\hline 108 & 57991.4194628627 & 1758.1366 & 121.3916 & 6230 & 1900 & 0.0293 & 0.0074 & 0.0295 & 0.015 & 1.43 & \\
\hline 109 & 57991.4208678576 & 1758.1380 & 29.8121 & 7110 & 1300 & 0.0391 & 0.0061 & 0.0286 & 0.0123 & 1.43 & \\
\hline 110 & 57991.4212129047 & 1758.1384 & 43.1795 & 7110 & 4000 & 0.1268 & 0.0106 & 0.0607 & 0.0151 & 0.72 & \\
\hline 111 & 57991.4217126673 & 1758.1389 & 35.3103 & 5670 & 3900 & 0.1858 & 0.0106 & 0.0785 & 0.0151 & 0.72 & \\
\hline 112 & 57991.4221213510 & 1758.1393 & 1.3489 & 5720 & 2000 & 0.064 & 0.0075 & 0.0682 & 0.017 & 1.79 & \\
\hline 113 & 57991.4221369638 & 1758.1393 & 69.3354 & 6900 & 2400 & 0.0557 & 0.0082 & 0.0397 & 0.0187 & 1.79 & \\
\hline 114 & 57991.4229394568 & 1758.1401 & 5.6751 & 6200 & 3900 & 0.2147 & 0.0105 & 0.1312 & 0.0238 & 1.79 & \\
\hline 115 & 57991.4230051411 & 1758.1402 & 109.3405 & 5770 & 4000 & 0.0355 & 0.0106 & 0.0263 & 0.0263 & 2.15 & \\
\hline 116 & 57991.4242706565 & 1758.1414 & 23.1794 & 6230 & 1900 & 0.0456 & 0.0074 & 0.053 & 0.0168 & 1.79 & \\
\hline 117 & 57991.4245389366 & 1758.1417 & 119.2321 & 5250 & 900 & 0.0312 & 0.0051 & 0.0174 & 0.009 & 1.08 & \\
\hline 118 & 57991.4259189384 & 1758.1431 & 41.0301 & 5750 & 900 & 0.0245 & 0.0051 & 0.0287 & 0.0127 & 2.15 & \\
\hline 119 & 57991.4263938242 & 1758.1436 & 13.7109 & 6170 & 1900 & 0.0561 & 0.0074 & 0.0675 & 0.0183 & 2.15 & \\
\hline 120 & 57991.4265525154 & 1758.1437 & 158.9548 & 6170 & 3900 & 0.3922 & 0.0105 & 0.1565 & 0.0151 & 0.72 & \\
\hline
\end{tabular}


International Journal of Cosmology, Astronomy and Astrophysics

\begin{tabular}{|c|c|c|c|c|c|c|c|c|c|c|c|}
\hline 121 & 57991.4283922705 & 1758.1456 & 17.3030 & 7140 & 2400 & 0.0574 & 0.0082 & 0.0746 & 0.022 & 2.51 & \\
\hline 122 & 57991.4285925362 & 1758.1458 & 158.5758 & 4780 & 3900 & 0.063 & 0.0105 & 0.0362 & 0.0184 & 1.08 & \\
\hline 123 & 57991.4304279044 & 1758.1476 & 57.7644 & 5670 & 3900 & 0.0704 & 0.0106 & 0.051 & 0.0185 & 1.08 & \\
\hline 124 & 57991.4310964736 & 1758.1483 & 49.7042 & 6160 & 1400 & 0.0271 & 0.0064 & 0.0574 & 0.0193 & 3.23 & \\
\hline 125 & 57991.4316717532 & 1758.1488 & 26.1148 & 6080 & 2000 & 0.0424 & 0.0075 & 0.0412 & 0.0169 & 1.79 & \\
\hline 126 & 57991.4319740079 & 1758.1491 & 26.0491 & 5660 & 3900 & 0.2084 & 0.0105 & 0.1629 & 0.0185 & 1.08 & \\
\hline 127 & 57991.4322755018 & 1758.1494 & 221.1596 & 5520 & 2000 & 0.058 & 0.0075 & 0.048 & 0.0151 & 1.43 & \\
\hline 128 & 57991.4348352197 & 1758.1520 & 31.3033 & 5730 & 1900 & 0.0288 & 0.0074 & 0.0243 & 0.0167 & 1.79 & \\
\hline 129 & 57991.4351975268 & 1758.1524 & 117.4965 & 7090 & 1900 & 0.0614 & 0.0073 & 0.0561 & 0.0149 & 1.43 & \\
\hline 130 & 57991.4365574396 & 1758.1537 & 111.6369 & 7120 & 1900 & 0.0715 & 0.0073 & 0.0564 & 0.0128 & 1.08 & \\
\hline 131 & 57991.4378495339 & 1758.1550 & 55.2223 & 6910 & 2300 & 0.041 & 0.0082 & 0.0244 & 0.0144 & 1.08 & \\
\hline 132 & 57991.4384886807 & 1758.1557 & 75.3406 & 5520 & 1500 & 0.042 & 0.0064 & 0.0224 & 0.0113 & 1.08 & \\
\hline 133 & 57991.4393606779 & 1758.1565 & 591.9158 & 7110 & 4000 & 0.2362 & 0.0106 & 0.2342 & 0.0239 & 1.79 & \\
\hline 134 & 57991.4462115554 & 1758.1634 & 67.1154 & 6160 & 1400 & 0.0387 & 0.0064 & 0.018 & 0.0091 & 0.72 & \\
\hline 135 & 57991.4469883544 & 1758.1642 & 81.2813 & 6440 & 1500 & 0.0395 & 0.0065 & 0.0131 & 0.0093 & 0.72 & \\
\hline 136 & 57991.4479291105 & 1758.1651 & 10.2570 & 5580 & 2900 & 0.0334 & 0.0091 & 0.0523 & 0.0244 & 2.51 & \\
\hline 137 & 57991.4480478257 & 1758.1652 & 32.8169 & 4970 & 900 & 0.0179 & 0.0051 & 0.0258 & 0.0128 & 2.15 & \\
\hline 138 & 57991.4484276509 & 1758.1656 & 151.8190 & 5580 & 3900 & 0.21 & 0.0105 & 0.1375 & 0.0184 & 1.08 & \\
\hline 139 & 57991.4501848147 & 1758.1673 & 24.4345 & 5390 & 1400 & 0.0463 & 0.0063 & 0.0197 & 0.009 & 0.72 & \\
\hline 140 & 57991.4504676214 & 1758.1676 & 222.2426 & 7470 & 2300 & 0.0405 & 0.0081 & 0.0343 & 0.0164 & 1.43 & \\
\hline 141 & 57991.4530398739 & 1758.1702 & 472.8758 & 4780 & 2400 & 0.0843 & 0.0083 & 0.0672 & 0.0168 & 1.43 & \\
\hline 142 & 57991.4585129735 & 1758.1757 & 203.4843 & 5530 & 1400 & 0.029 & 0.0064 & 0.0218 & 0.0144 & 1.79 & \\
\hline 143 & 57991.4608681160 & 1758.1780 & 245.9356 & 7530 & 1400 & 0.054 & 0.0063 & 0.0272 & 0.011 & 1.08 & \\
\hline 144 & 57991.4637145928 & 1758.1809 & 19.4120 & 6750 & 1900 & 0.0731 & 0.0074 & 0.0471 & 0.013 & 1.08 & \\
\hline 145 & 57991.4639392697 & 1758.1811 & 0.0024 & 5480 & 3000 & 0.0573 & 0.0092 & 0.0367 & 0.0161 & 1.08 & \\
\hline 146 & 57991.4639392978 & 1758.1811 & 539.7461 & 5480 & 2000 & 0.0388 & 0.0075 & 0.0439 & 0.0152 & 1.43 & \\
\hline 147 & 57991.4701863590 & 1758.1873 & 82.9205 & 6220 & 1400 & 0.0452 & 0.0064 & 0.0205 & 0.0091 & 0.72 & \\
\hline 148 & 57991.4711460870 & 1758.1883 & 149.1976 & 6620 & 1500 & 0.0308 & 0.0065 & 0.0272 & 0.0161 & 2.15 & \\
\hline 149 & 57991.4728729109 & 1758.1900 & 618.5275 & 5060 & 900 & 0.0255 & 0.0051 & 0.0201 & 0.0115 & 1.79 & \\
\hline 150 & 57991.4800317945 & 1758.1972 & 740.5645 & 4830 & 2000 & 0.0469 & 0.0075 & 0.0353 & 0.0169 & 1.79 & \\
\hline 151 & 57991.4886031426 & 1758.2058 & 393.6307 & 6420 & 900 & 0.0223 & 0.0051 & 0.0265 & 0.0145 & 2.87 & \\
\hline 152 & 57991.4931590534 & 1758.2103 & 375.8981 & 5110 & 1900 & 0.0266 & 0.0073 & 0.0185 & 0.0166 & 1.79 & \\
\hline 153 & 57991.4975097257 & 1758.2147 & 75.7672 & 4830 & 2000 & 0.0681 & 0.0075 & 0.05 & 0.0151 & 1.43 & \\
\hline 154 & 57991.4983866613 & 1758.2155 & 37.0514 & 5940 & 900 & 0.0268 & 0.0051 & 0.0246 & 0.0127 & 2.15 & \\
\hline 155 & 57991.4988154972 & 1758.2160 & 1040.3803 & 5530 & 2400 & 0.0417 & 0.0083 & 0.0515 & 0.0187 & 1.79 & \\
\hline 156 & 57991.5108569357 & 1758.2280 & 194.5233 & 7330 & 2000 & 0.0555 & 0.0075 & 0.0241 & 0.0107 & 0.72 & \\
\hline 157 & 57991.5131083628 & 1758.2303 & 528.9577 & 4830 & 2000 & 0.1284 & 0.0075 & 0.0964 & 0.0131 & 1.08 & \\
\hline 158 & 57991.5192305588 & 1758.2364 & 0.0028 & 6910 & 2000 & 0.1106 & 0.0075 & 0.0498 & 0.0215 & 2.87 & \\
\hline 159 & 57991.5192305898 & 1758.2364 & 3470.2810 & 6910 & 2000 & 0.0988 & 0.0075 & 0.0659 & 0.0152 & 1.43 & \\
\hline 160 & 57991.5593958795 & 1758.2766 & 295.8331 & 6980 & 3900 & 0.0765 & 0.0105 & 0.0297 & 0.0151 & 0.72 & \\
\hline 161 & 57991.5628198735 & 1758.2800 & 572.6219 & 6830 & 2800 & 0.1022 & 0.009 & 0.0585 & 0.0157 & 1.08 & \\
\hline 162 & 57991.5694474415 & 1758.2866 & 134.2970 & 5950 & 2000 & 0.0448 & 0.0075 & 0.0696 & 0.0214 & 2.87 & \\
\hline 163 & 57991.5710018046 & 1758.2882 & 297.4788 & 5670 & 1900 & 0.0434 & 0.0074 & 0.0492 & 0.0149 & 1.43 & \\
\hline 164 & 57991.5744448464 & 1758.2916 & 1184.0859 & 6420 & 900 & 0.0183 & 0.0051 & 0.0177 & 0.0136 & 2.51 & \\
\hline 165 & 57991.5881495443 & 1758.3053 & 204.0394 & 5580 & 2000 & 0.0679 & 0.0075 & 0.0478 & 0.0131 & 1.08 & \\
\hline 166 & 57991.5905111113 & 1758.3077 & 138.2585 & 6410 & 2400 & 0.0644 & 0.0083 & 0.0421 & 0.0146 & 1.08 & \\
\hline 167 & 57991.5921113250 & 1758.3093 & 286.5264 & 5940 & 2000 & 0.0862 & 0.0075 & 0.0518 & 0.0131 & 1.08 & \\
\hline 168 & 57991.5954276026 & 1758.3126 & 28.6749 & 6250 & 1500 & 0.059 & 0.0065 & 0.0209 & 0.0093 & 0.72 & \\
\hline 169 & 57991.5957594875 & 1758.3129 & 561.9668 & 6250 & 1500 & 0.0329 & 0.0065 & 0.0292 & 0.0131 & 1.43 & \\
\hline 170 & 57991.6022637323 & 1758.3194 & & 4920 & 1300 & 0.0601 & 0.0061 & 0.0213 & 0.0087 & 0.72 & \\
\hline 171 & 57996.2656372 & 1762.9828 & & 1400 & 300 & 24 & 7 & 35 & 10 & 2.6 & 0.40 \\
\hline
\end{tabular}

Note: BPT: Barycentric peak time; TOA: time of arrival; WT: waiting time. S: peak flux; F: fluence. w: width, or the duration of the burst. For the references of the observational data, please see (Table 1).

\section{Statistical Characteristics of Waiting Times}

We use the available waiting time data for statistical analysis. The distribution of the waiting time is plotted as a histogram in (Figure 1). It can be clearly seen that there is a bimodal distribution. A small portion of the waiting times cluster in the range of 0.002-0.04 s. A Gaussian fit for them gives the peak time as $\Delta t \approx 0.0015 \mathrm{~s}$. It is very striking that FRBs could repeat after such a short quiescent period.
However, note that due to very limited number of events, significant fluctuation could be seen in the fit. In figure 1 , most of the waiting times lie in the range of 1-6100 s. They can be well fitted by another Gaussian function with a reduced Chi-Sqr 6.259 and aAdj. R-Square of $~ 0.945$. The peak value of the waiting time is $\Delta t=169.93$ for these events. In our plot, since the $\mathrm{X}$-axis is the logarithm of $\Delta \mathrm{t}$, the Gaussian function means these waiting times follow a Log-normal distribution. 


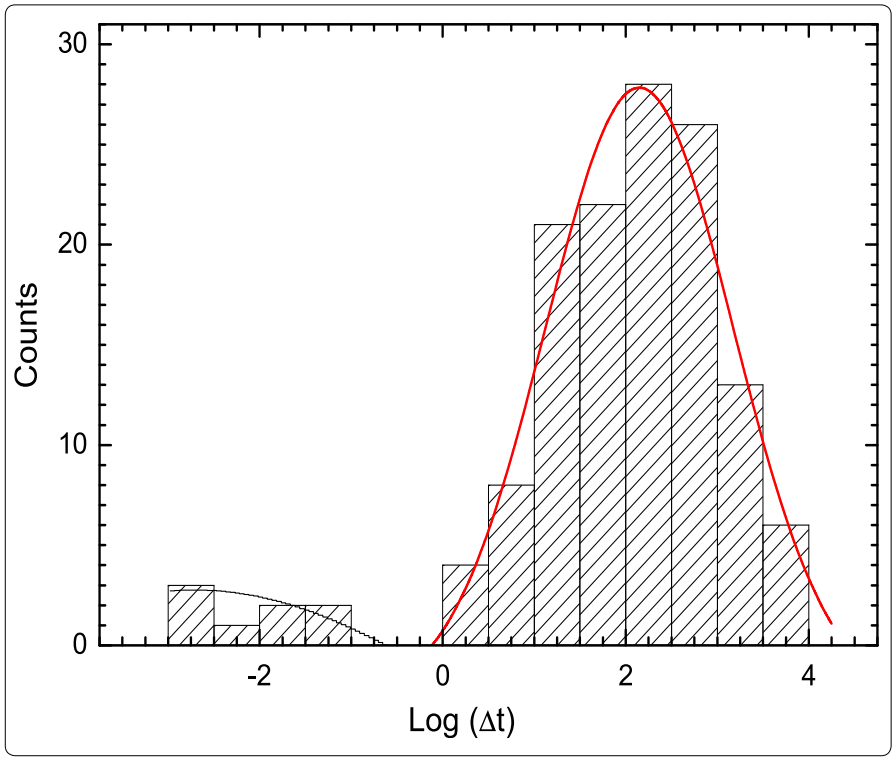

Figure 1. A histogram plot of the distribution of the waiting time ( $\Delta \mathrm{t}$, in units of s). The $\mathrm{X}$-axis of the $\Delta \mathrm{t}$ data was logarithm, and the bin size is 0.5 . A clear bimodal distribution can be seen. Most of the waiting times cluster at around several hundred seconds. They can

be well fit by a Gaussian function, which means they follow a

Log-normal distribution (the red line). A small portion of the waiting times are far below 1 millisecond. They still could be fit with a Gaussian function (the black line), but with significant fluctuation.

The cumulative distribution of the waiting time is shown in the (Figure 2). In this figure, the red line shows all the calculated waiting time data in (Table 2). However, since the bursts were observed at different frequencies by different receivers with different sensitivities, we have also plot the six sub-sets of data in the figure, i.e. GBT/C-band $(4-8 \mathrm{GHz}), \mathrm{GBT} /$ S-band (1.6-2.4 GHz), AO/C-band $(4.1-4.9 \mathrm{GHz}), \mathrm{AO} / \mathrm{L}-$ band and ALFA (1.2-1.5 GHz) and Eff/L-band (1.2-1.5 GHz), respectively. Generally speaking, these plots give us a straightforward vision of the effect of the telescope sensitivity. Due to the more efficient search technique of machine learning $[99,101]$, the contribution of the observation campaign at GBT/C-band is very striking.

According to some models, the energy released in an FRB burst are dependent on the quiescent period between two consecutive bursts, i.e., the waiting time. This is especially true for FRB models in which some kinds of intrinsic mechanisms are involved, such as the star quake models, the giant flare models of magnetars, the accretion-induced collapse of the crust of neutron stars or strange stars, etc. The reason is obvious: it is usually necessary for the system to accumulate some amount of energy to give birth to the next burst. As a result, the intensity of the burst should be correlated with the waiting time. On the contrary, in most models involving external mechanisms, no such correlation is expected. For example, if the FRB is produced by the collision of a small body or by many small bodies in an asteroid belt [62-64], the burst intensity will not correlate with the waiting time. So, the relationship between the burst intensity and the waiting time can provide valuable clues on the FRB models.

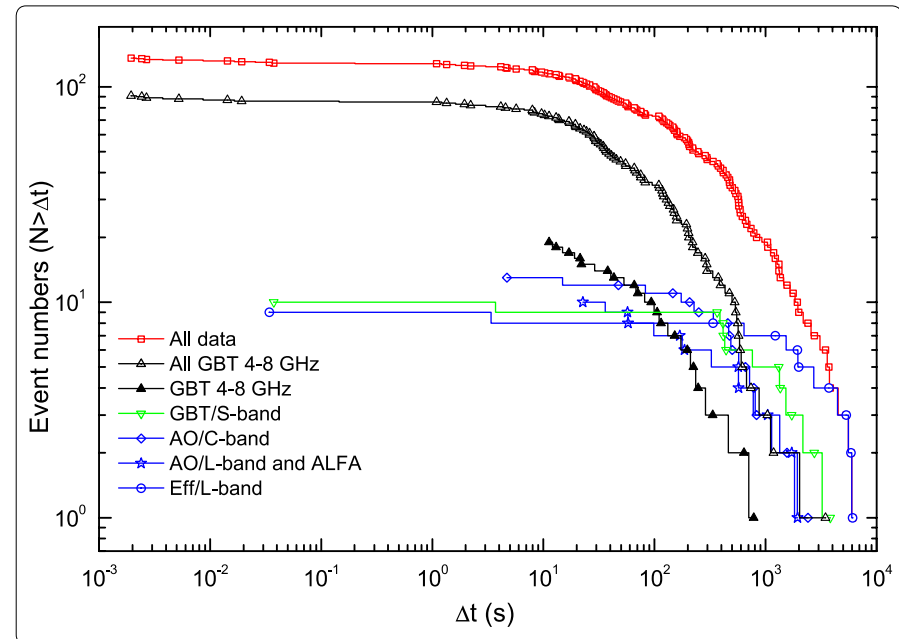

Figure 2. Cumulative distribution of the waiting time $(\Delta t)$. The hollow red squares show all the waiting time available for FRB 121102, as listed in Table II. Other plots correspond to various sub-set of the data, i.e., the All GBT/4-8 GHz data (hollow black triangles) [101], part of the GBT/4-8 GHz data (filled black triangles) [99], the GBT/S-band data (opposite hollow triangles in green color) $[93,97]$, the $\mathrm{AO} / \mathrm{C}$-band data (hollow diamonds in blue) [77], the AO/L-band and ALFA data (hollow stars in blue) [92, 97], the Eff/L-band data (hollow circulars in blue) [94].

We have used our waiting time data to try to search for any possible correlation between the waiting time and the burst intensity. However, before presenting the results, it should be further noted that the waiting time actually could be correlated with either the preceding burst or the subsequent burst. We thus need to check these two cases separately.

In Figure 3, we plot the waiting time versus some interesting parameters, such as the peak flux density (Speak), the duration (the Gaussian width, w), the fluence ( $F$, which is calculated by multiplying Speak and w). Form figure 3, we can see the data points are widely scattered in all the plots, so that no obvious correlation can be observed between the burst intensity and the waiting time. This feature can provide us important clues about the nature of the repeating FRB source.

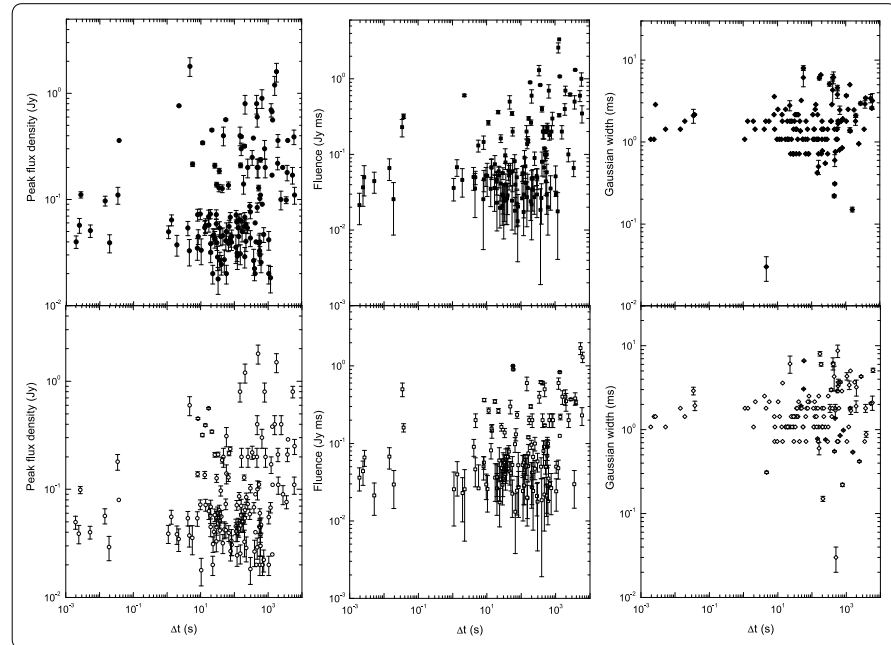

Figure 3. Waiting time $(\Delta t)$ versus other three parameters of FRB bursts, i.e., the peak flux density (the left panels), the fluence (the middle panels), and the width (the right panels). In the upper three panels, the parameters are for the preceding bursts, while in the bottom three panels, the parameters are for the subsequent bursts. In all the plots, no obvious correlation can be seen between the three parameters and the waiting time. 
As mentioned in Section $\mathrm{l}$, all the FRB models proposed in the literature can be grouped into two different categories, i.e. catastrophic models and non-catastrophic models. For FRB 121102, surely only the non-catastrophic models can meet its basic requirement of repetition. It is interesting to note that the non-catastrophic models again can have two classes: those due to internal mechanisms and those due to external mechanisms. For example, most of the noncatastrophic models such as star quake-like explosions, flaring stars, lightning/wandering in pulsars, giant pulses/flares from young pulsars, collapse of strange star crusts, are all mechanisms involving internal processes. In these cases, the energy release is mainly caused by magnetic reconnection or accretion. So, to prepare for a new burst event, it usually takes a period of time to accumulate the energy. As a result, there is usually a positive correlation between the waiting time and the burst energetics. On the contrary, models involving the collisions between small bodies and neutron stars [62-64] are the few kinds of non-catastrophic models in which external mechanisms are engaged. In these cases, the happening of the bursts is almost completely random. The system does not need to accumulate any intension for the next burst. Therefore, the nonexistence of any correlation between the waiting time and other parameters (especially with the burst energetics) as shown in our figure 3 seem to rule out many intrinsic mechanism models for FRBs, but strongly support the external mechanism models, especially the collisions between small bodies and neutron stars [62-64].

\section{Discussion and Conclusion}

FRB 121102 is the only source that is observed to burst out repeatedly till now. In this study, we analyze the repeating behavior of FRB 121102 statistically, paying special attention on the waiting time. It is found that the waiting time shows a clear bimodal distribution, clustering at 0.002-0.04 $\mathrm{s}$ and $170 \mathrm{~s}$ respectively. It is striking to note that some bursts could happen very shortly after the preceding burst. We also tried to examine any possible correlation between the waiting time and the burst intensity. It is found that the waiting time does not correlate with the intensity (characterized by the peak flux and the burst fluence) of either the preceding burst or the subsequent burst. This result strongly indicates that the repeating bursts should be produced by some external mechanisms, but not intrinsic mechanisms. We suggest that the models involving collisions between small bodies and neutron stars [62-64] could be competitive mechanisms for FRBs.

Currently, due to the occultation of the Earth, FRB 121102 could not be continuously monitored for too long in any observational campaign. Usually, it could be continuously monitored for only one or two hours, and in a few very rare cases, the monitoring period could be extended to several hours. As a result, it is difficult for us to derive the waiting time that is longer than several thousands. This may be the reason that there is a cutoff at the longer section of the waiting time distribution. It is suggested the various large telescopes could cooperate to carry out an all-time monitoring campaign on
FRB 121102. It can help to clarify the waiting time distribution in the longer period regime, and may be important for understanding the nature of this enigmatic FRB source.

The bursts of FRB 121102 shows a clustering behavior in time. There are many outbursts in some particular time periods, which leads to very short waiting time for these bursts. For example, six bursts (Bursts 6 to 11) arrived in less than twenty minutes' period and four bursts (Bursts 13 to 16) occurred in a $\sim 50$ minutes' period during the Observation Campaign 4. Even more prominent is on the 33th and 34th observation campaign, where more frequent eruptions were observed within half an hour. It indicates that there appears to be epochs in which the source is more active. However, it should also be pointed out that there are also several very long observing sessions which resulted in non-detections [93, $95,116]$. The clustering phenomenon has been discussed by a few authors [117]. For example, a neutron star traveling through an asteroid belt can naturally lead to an active period and give birth to such a phenomenon [62-64]. In this case, the next active period could even be forecasted [119]. However, since FRB 121102 has been discovered for only less than 8 years, it is still an open question whether the repeating activity shows any difference on larger time-scales [120].

As shown in our figure 1, the majority of the waiting times follow a log-Gaussian distribution. This behavior is very similar to a few activities observed in other celestial objects, such as the waiting times of hard X-ray bursts from the Sun [118], the waiting times of hard $X$-ray bursts from the famous soft gamma-ray repeater SGR 1900+14 [105], and the waiting times of the Crab pulsar glitches $\left(\sim 10^{2}\right.$ to $\left.\sim 10^{6} \mathrm{~s}\right)$ [107]. In all these cases, the waiting times follow similar log-normal distributions. The common reason may be that they are all random processes to some extent.

In the bimodal distribution of the waiting time, there are about ten bursts clustered in the region with the waiting time less than 1 second, most of which were found by using the new searching technique [101]. However, while a few of these bursts are weak, there are also some strong bursts among them. It is thus even more difficult for the intrinsic mechanisms to explain such a fact: how the central engine could produce so many strong bursts in such a short period. On the contrary, this issue can be naturally accounted for by an external mechanism such as the collision between small bodies with neutron stars. It is possible that when a neutron star travels through an asteroid belt, it may encounter several asteroids one after the other, producing a group of bursts in a short time.

\section{Acknowledgments}

This work is supported by the National Natural Science Foundation of China (Grant Nos. 11873030, 11473012, and U1431126), the National Postdoctoral Program for Innovative Talents (Grant No. BX201700115), China Postdoctoral Science Foundation funded project (Grant No. 2017M620199), Guizhou Provincial Natural Science Foundation (201519), and by the 
Strategic Priority Research Program of the Chinese Academy of Sciences "Multi-waveband Gravitational Wave Universe" (Grant No. XDB23040000 and XDB23040400). LMS acknowledges support from the National Program on Key Research and Development Project (Grant No. 2016YFA0400801). YPY is supported by the Initiative Postdocs Supporting Program (No. BX201600003) and the China Postdoctoral Science Foundation (No.2016M600851). BL is also supported by Key Research Program of Frontier Sciences, Chinese Academy of Sciences (Grant NO. QYZDB-SSW-SLH012).

\section{References}

1. Lorimer DR, Bailes M, McLaughlin MA, Narkevic DJ, Crawford F. A Bright Millisecond Radio Burst of Extragalactic Origin. Science. 2007; 318(5851): 777-780. doi: 10.1126/science. 1147532

2. Petroff E, Barr ED, Jameson A, et al. FRBCAT: The Fast Radio Burst Catalogue. Publications of the Astronomical Society of Australia. 2016; 33: 45-52. doi: 10.1017/pasa.2016.35

3. Thornton D, Stappers B, Bailes M. A Population of Fast Radio Bursts at Cosmological Distances. Science. 2013; 341(6141): 53-56. doi: 10.1126/ science. 1236789

4. Champion DJ, Petroff E, Kramer M, et al. Five new fast radio bursts from the HTRU high-latitude survey at Parkes: first evidence for two-component bursts. MNRAS. 2016; 460(1): L30-L34. doi: 10.1093/mnrasl/slw069

5. Cordes JM, Wharton RS, Spitler LG, Chatterjee S, Wasserman G. Radio Wave Propagation and the Provenance of Fast Radio Bursts. High Energy Astrophysical Phenomena. 2018; 1605: 05890.

6. Bannister KW, Shannon RM, Macquart JP, et al. The Detection of an Extremely Bright Fast Radio Burst in a Phased Array Feed Survey. ApJL. 2017; 841(1): L12-L18. doi: 10.3847/2041-8213/aa71ff

7. Yao JM, Manchester RN, Wang N. A New Electron-density Model for Estimation of Pulsar and FRB Distances. ApJ. 2017; 835: 29-78. doi: 10.3847/1538-4357/835/1/29

8. Caleb M, Keane EF, van Straten W, et al. The SUrvey for Pulsars and Extragalactic Radio Bursts - III. Polarization properties of FRBs 160102 and 151230. MNRAS. 2018; 478(2): 2046-2055. doi: 10.1093/mnras/sty1137

9. Shannon RM, Macquart JP, Bannister KW, et al. The dispersion-brightness relation for fast radio bursts from a wide-field survey. Nature. 2018; 562: 386-390. doi: 10.1038/s41586-018-0588-y

10. Ravi V. The observed properties of fast radio bursts. MNRAS. 2019; 482(2): 1966-1978. doi: 10.1093/mnras/sty1551

11. Ravi V, Shannon RM, Bailes $\mathrm{M}$, et al. The magnetic field and turbulence of the cosmic web measured using a brilliant fast radio burst. Science. 2016; 354: 1249-1252. doi: 10.1126/science.aaf6807

12. Petroff $E$, Oostrum LC, Stappers BW, et al. A fast radio burst with a low dispersion measure. MNRAS. 2019; 482(3): 3109-3115. doi: 10.1093/ mnras/sty2909

13. Patel $C$, Agarwal $D$, Bhardwaj $M$, et al. PALFA Single-Pulse Pipeline: New Pulsars, Rotating Radio Transients and a Candidate Fast Radio Burst. APJ. 2018.

14. Dolag K, Gaensler BM, Beck AM, Beck MC. Constraints on the distribution and energetics of fast radio burstsusing cosmological hydrodynamic simulations. MNRAS. 2015; 451(4): 4277-4289. doi: 10.1093/mnras/stv1190

15. Caleb $M$, Flynn $C$, Bailes $M$, et al. Are the distributions of fast radio burst properties consistent with a cosmological population? MNRAS. 2016; 458(1): 708-717. doi: 10.1093/mnras/stw175

16. Katz Jl. Inferences from the Distributions of Fast Radio Burst Pulse Widths, Dispersion Measures, and Fluences. ApJ. 2016; 818: 19-28. doi: 10.3847/0004$637 \mathrm{X} / 818 / 1 / 19$

17. Yang $Y P$, Luo R, Li Z, Zhang B. Large Host-galaxy Dispersion Measure of Fast Radio Bursts. ApJL. 2017; 839: L25-L30. doi: 10.3847/2041-8213/aa6c2e
18. Wei JJ, Wu XF, Gao H. Cosmology with Gravitational Wave/Fast Radio Burst Associations. ApJL. 2018; 860: L7-L13. doi: 10.3847/2041-8213/aac8e2

19. Ghisellini $G$, Locatelli $N$. Coherent curvature radiation and fast radio bursts. A\&A. 2018; 613: 61-69. doi: 10.1051/0004-6361/201731820

20. Katz Jl. Coherent plasma-curvature radiation in FRB. MNRAS. 2018; 481: 2946-2950. doi: 10.1093/mnras/sty2459

21. Yang YP, Zhang B. Bunching Coherent Curvature Radiation in Threedimensional Magnetic Field Geometry: Application to Pulsars and Fast Radio Bursts. ApJ. 2018; 868: 31-50. doi: 10.3847/1538-4357/aae685

22. Lyubarsky Y. A model for fast extragalactic radio bursts. MNRAS. 2014; 442: L9-L13. doi: 10.1093/mnrasl/slu046

23. Ghisellini G. Synchrotron masers and fast radio bursts. MNRAS. 2017; 465: L30-L33. doi: 10.1093/mnrasl/slw202

24. Waxman E. On the Origin of Fast Radio Bursts (FRBs). ApJ. 2017; 842: 3442. doi: 10.3847/1538-4357/aa713e

25. Long K, Pe'er A. Synchrotron maser from weakly magnetized neutron stars as the emission mechanism of fast radiobursts. ApJ. 2018.

26. Lu W, Kumar P. On the radiation mechanism of repeating fast radio bursts. 2018; 477: 2470-2493. doi: 10.1093/mnras/sty716

27. Katz J. Fast radio bursts. Progress in Particle and Nuclear Physics. 2018; 103: 1-18. doi: 10.1016/j.ppnp.2018.07.001

28. Caleb M, Spitler LG, Stappers BW. One or several populations of fast radio burst sources? Nature Astronomy. 2018; 2: 839-841. doi: 10.1038/s41550018-0612-z

29. Pen UL. The nature of fast radio bursts. Nature Astronomy. 2018; 2: 842844. doi: 10.1038/s41550-018-0620-z

30. Totani T. Cosmological Fast Radio Bursts from Binary Neutron Star Mergers. PASJ. 2013; 65(5): L12-L16. doi: 10.1093/pasj/65.5.L12

31. Wang JS, Yang YP, Wu XF, Dai ZG, Wang FY. Fast Radio Bursts from the Inspiral of Double Neutron Stars. ApJL. 2016; 822: L7-L12. doi: 10.3847/2041-8205/822/1/L7

32. Dokuchaev VI, Eroshenko YN. Recurrent fast radio bursts from collisions of neutron stars in the evolved stellar clusters. ApJ. 2017.

33. Kashiyama K, Oka K, Meszros P. Cosmological Fast Radio Bursts from Binary White Dwarf Mergers. ApJL. 2013; 776: L39-L43. doi: 10.1088/20418205/776/2/L39

34. Gu WM, Dong YZ, Liu T, Ma R, Wang J. A Neutron Star-White Dwarf Binary Model for Repeating Fast Radio Burst 121102. ApJL. 2016; L28-L33. doi: 10.3847/2041-8205/823/2/L28

35. Liu X. A model of neutron-star-white-dwarf collision for fast radio bursts Ap\&SS. 2018; 363: 242-250. doi: 10.1007/s10509-018-3462-3

36. Mingarelli CMF, Levin J, Lazio TJW. Fast Radio Bursts and Radio Transients from Black Hole Batteries. ApJL. 2015; 814: L20-L25. doi: 10.1088/2041$8205 / 814 / 2 / L 20$

37. Abramowicz MA, Bejger M, Wielgus M. Collisions of Neutron Stars with Primordial Black Holes as Fast Radio Bursts Engines. ApJ. 2018; 868: 17-24. doi: 10.3847/1538-4357/aae64a

38. Li LB, Huang YF, Geng JJ, Li B. A model of fast radio bursts: collisions between episodic magnetic blobs. Research in Astronomy and Astrophysics. 2018; 18: 061-067. doi: 10.1088/1674-4527/18/6/61

39. Bhattacharyya S. Fast Radio Bursts from neutron stars plunging into black holes. ApJ. 2017.

40. Liu T, Romero GE, Liu ML, Li A. Fast Radio Bursts and Their Gamma-Ray or Radio Afterglows as Kerr-Newman Black Hole Binaries. ApJ. 2016; 826: 82-88. doi: 10.3847/0004-637X/826/1/82

41. Zhang Bing. Mergers of Charged Black Holes: Gravitational Wave Events, Short Gamma-Ray Bursts, and Fast Radio Bursts. ApJL. 2016; 827(2): L31-L36. doi: 10.3847/2041-8205/827/2/L31

42. Falcke $\mathrm{H}$, Rezzolla L. Fast radio bursts: the last sign of supramassive neutron stars. A\&A. 2014; 562: A137-A143. doi: 10.1051/0004-6361/201321996 
43. Zhang B. A possible connection between Fast Radio Bursts and GammaRay Bursts. ApJL. 2014; 780: L21-L25. doi: 10.1088/2041-8205/780/2/L21

44. Fuller J, Ott CD. Dark matter-induced collapse of neutron stars: a possible link between fast radio bursts and the missing pulsar problem. MNRAS. 2015; 450: L71-L75. doi: 10.1093/mnrasl/slv049

45. Punsly B, Bini D. General relativistic considerations of the field shedding model of fast radio bursts. MNRAS. 2016; 459. L41-L45. doi: 10.1093/ mnrasl/slw039

46. Shand Z, Ouyed A, Koning N, Ouyed R. Quark nova model for fast radio bursts. Research in Astronomy and Astrophysics. 2016; 16: 80-92. doi: 10.1088/1674-4527/16/5/080

47. Zhang Y, Geng JJ, Huang YF. Fast Radio Bursts from the Collapse of Strange Star Crusts. ApJ. 2018; 858: 88-94. doi: 10.3847/1538-4357/ aabaee

48. Keane EF, Stappers BW, Kramer M, Lyne AG. On the origin of a highly dispersed coherent radio burst. MNRAS. 2012; 425: L71-L75. doi: 10.1111/j.1745-3933.2012.01306.x

49. Cordes JM, Wasserman I. Supergiant pulses from extragalactic neutron stars. MNRAS. 2016; 457: 232-257. doi: 10.1093/mnras/stv2948

50. Connor L, Sievers J, Pen UL. Non-cosmological FRBs from young supernova remnant pulsars. MNRAS. 2016; 458: L19-L23. doi: 10.1093/ mnrasl/slv124

51. Lieu R. Are Fast Radio Bursts the Birthmark of Magnetars? ApJ. 2017; 834: 199-214. doi: 10.3847/1538-4357/834/2/199

52. Popov SB, Postnov KA. Hyperflares of SGRs as an engine for millisecond extragalactic radio bursts. MNRAS. 2007.

53. Popov SB, Postnov KA. Millisecond extragalactic radio bursts as magnetar flares. MNRAS. 2013

54. Murase K, Kashiyama K, Meszaros P. A burst in a wind bubble and the impact on baryonic ejecta: high-energygamma-ray flashes and afterglows from fast radio bursts and pulsar-driven supernova remnants. MNRAS. 2016; 461: 1498. doi: 10.1093/mnras/stw1328 10.1093/mnras/stx310

55. Beloborodov AM. Flaring Magnetar in FRB 121102? ApJ. 2017; 843(2): L26-L31. doi: 10.3847/2041-8213/aa78f3

56. Romero GE, del Valle MV, Vieyro FL. Mechanism for fast radio bursts. Phys. Rev. D. 2016; 93(2): 023001-023008. doi: 10.1103/PhysRevD.93.023001

57. Vieyro FL, Romero GE, Bosch-Ramon V, Marcote B, del Valle MV. A model for the repeating FRB 121102 in the AGN scenario. A\&A. 2017; 602: A64-A71. doi: 10.1051/0004-6361/201730556

58. Das Gupta P, Saini N. Collapsing supra-massive magnetars: FRBs, the repeating FRB121102 and GRBs. ApJ. 2018; 39: 185.

59. Katz Jl. FRB as products of accretion disc funnels. MNRAS. 2017; 471: L92-L95. doi: 10.1093/mnrasl/slx113

60. Yue Z, Jun GJ, Feng HY. Fast Radio Bursts from the collapse of Strange Star Crusts. ApJL. 2018; 858(2): 88-94. doi: 10.3847/1538-4357/aabaee

61. Mottez F, Zarka P. Radio emissions from pulsar companions: a refutable explanation for galactic transients and fast radio bursts. A\&A. 2014; 569; A86-97. doi: 10.1051/0004-6361/201424104

62. Geng JJ, Huang YF. Fast Radio Bursts: Collisions between Neutron Stars and Asteroids/Comets. ApJ. 2015; 809: 24-29. doi: 10.1088/0004$637 \mathrm{X} / 809 / 1 / 24$

63. Huang YF, Geng JJ. Collision between Neutron Stars and Asteroids as a Mechanism for Fast Radio Bursts. Frontiers in Radio Astronomy and FAST Early Sciences Symposium 2015. Proceedings of a Workshop held at Guiyang China 29-31 July 2015. Astronomical Society of the Pacific. 2016; 502: 1-6.

64. Dai ZG, Wang JS, Wu XF, Huang YF. Repeating Fast Radio Bursts from Highly Magnetized Pulsars Traveling through Asteroid Belts. ApJ. 2016; 829: 27-34. doi: 10.3847/0004-637X/829/1/27

65. Aiichi I. Axion stars and fast radio bursts. Phys. Rev. D. 2015; 91: 023008. doi: 10.1103/PhysRevD.91.023008
66. Stuart R. Axion star collisions with neutron stars and fast radio bursts. Phys. Rev. D. 2016; 94: 103004. doi: 10.1103/PhysRevD.94.103004

67. Aiichi I. Axion Stars and Repeating Fast Radio Bursts with Finite Bandwidths. ApJ. 2017.

68. Tkachev II. Fast Radio Bursts and Axion Miniclusters. Soviet Journal of Experimental and Theoretical Physics Letters. 2015; 101(1): 1-6. doi: 10.1134/S0021364015010154

69. Rosa, JG, Kephart TW. Stimulated Axion Decay in Superradiant Clouds around Primordial Black Holes. Phys. Rev. Lett. 2018; 120(23): 231102. doi: 10.1103/PhysRevLett.120.231102

70. Ludovic VW, Ariel Z. Fast Radio Bursts and the Axion Quark Nugget Dark Matter Model. ApJ. 2018.

71. Wang W, Luo R, Yue H, Chen X, Lee K, Xu R. FRB 121102: A Starquakeinduced Repeater? ApJ. 2018; 140-146. doi: 10.3847/1538-4357/aaa025

72. Barrau A, Rovelli C, Vidotto F. Fast Radio Bursts and White Hole Signals. Phys. Rev. D. 2014; 90(12): 127503. doi: 10.1103/PhysRevD.90.127503

73. Barrau A, Moulin F, Martineau K. Fast radio bursts and the stochastic lifetime of black holes in quantum gravity. Phys. Rev. D. 2018; 97(6) 066019. doi: 10.1103/PhysRevD.97.066019

74. Katz JI. Fast radio bursts as pulsar lightning. MNRAS. 2017; 469(1): L39-L42. doi: 10.1093/mnrasl/slx052

75. Bing Z. A "Cosmic Comb" Model of Fast Radio Bursts. ApJL. 2017; 836(2): L32-L36. doi: 10.3847/2041-8213/aa5ded

76. Bing Z. FRB 121102: A Repeatedly Combed Neutron Star by a Nearby Low-luminosity Accreting Supermassive Black Hole. ApJL. 2018; 854(2): L21-L26. doi: 10.3847/2041-8213/aaadba

77. Michilli D, Seymour A, Hessels JWT, et al. An extreme magneto-ionic environment associated with the fast radio burst source FRB 121102 Nature. 2018; 553: 182-185. doi: 10.1038/nature25149

78. Palaniswamy D, Li Y, Zhang B. Are There Multiple Populations of Fast Radio Bursts? ApJL. 2018; 854: L12. doi: 10.3847/2041-8213/aaaa63

79. Rane A, Lorimer D. Fast Radio Bursts. Journal of Astrophysics and Astronomy. 2017; 38: 55-68. doi: 10.1007/s12036-017-9478-1

80. Platts E, Weltman A, Walters A, et al. A Living Theory Catalogue for Fast Radio Bursts. ApJl. 2018

81. Dai ZG, Wang JS, Yu YW. Radio Emission from Pulsar Wind Nebulae without Surrounding Supernova Ejecta:Application to FRB 121102. ApJL. 2017; 838: L7-L12. doi: 10.3847/2041-8213/aa6745

82. Li LB, Huang YF, Geng JJ. Progress in the Researches of Fast Radio Bursts. Chinese Astronomy and Astrophysics. 2018; 42(2): 165-187. doi: 10.1016/j. chinastron.2018.04.001

83. Petroff $E$, Bailes $M$, Barr $E D$, et al. A real-time fast radio burst: polarization detection and multiwavelength follow-up. MNRAS. 2015; 447: 246-255. doi: 10.1093/mnras/stu2419

84. Petroff E, Burke-Spolaor $\mathrm{S}$, Keane EF, et al. A polarized fast radio burst at low Galactic latitude. MNRAS. 2017; 469: 4465-4482. doi: 10.1093/mnras/ stx 1098

85. Bhandari S, Keane EF, Barr ED, et al. The SUrvey for Pulsars and Extragalactic Radio Bursts - II. New FRB discoveries and their follow-up. MNRAS. 2018; 475: 1427-1446. doi: 10.1093/mnras/stx3074

86. Ravi V, Shannon RM, Jameson A. A Fast Radio Burst in the Direction of the Carina Dwarf Spheroidal Galaxy. ApJL. 2015; 799: L5-L20. doi: 10.1088/2041-8205/799/1/L5

87. Spitler LG, Cordes JM, Hessels JWT, et al. Fast Radio Burst Discovered in the Arecibo Pulsar ALFA Survey. ApJ. 2014; 790: 101-110. doi: 10.1088/0004-637X/790/2/101

88. Chatterjee S, Law CJ, Wharton RS, et al. A direct localization of a fast radio burst and its host. Nature. 2017; 541: 58-61. doi: 10.1038/nature20797

89. Bassa CG, Tendulkar SP, Adams EAK, et al. FRB 121102 Is Coincident with a Star-forming Region in Its Host Galaxy. ApJL. 2017; 843: L8-L14. doi: 10.3847/2041-8213/aa7a0c 
90. Marcote B, Paragi Z, Hessels JWT, et al. The Repeating Fast Radio Burst FRB 121102 as Seen on Milliarcsecond Angular Scales. ApJL. 2017; 834: L8-L17. doi: 10.3847/2041-8213/834/2/L8

91. Tendulkar SP, Bassa CG, Cordes JM, et al. The Host Galaxy and Redshift of the Repeating Fast Radio Burst FRB 121102. ApJL. 2017; 834: L7L-L15. doi: 10.3847/2041-8213/834/2/L7

92. Spitler $L G$, Scholz $P$, Hessels JWT, et al. A repeating fast radio burst. Nature. 2016; 531: 202-205. doi: 10.1038/nature17168

93. Scholz P, Spitler LG, Hessels JWT, et al. The Repeating Fast Radio Burst FRB 121102: Multi-wavelength Observations and Additional Bursts. ApJ. 2016; 833: 177-194. doi: 10.3847/1538-4357/833/2/177

94. Hardy LK, Dhillon VS, Spitler LG. A search for optical bursts from the repeating fast radio burst FRB 121102. MNRAS. 2017; 472: 2800-2807. doi: 10.1093/mnras/stx2153

95. Law CJ, Abruzzo MW, Bassa CG, et al. A Multi-telescope Campaign on FRB 121102: Implications for the FRB Population. ApJ. 2017; 850: 76-90. doi: 10.3847/1538-4357/aa9700

96. Oostrum LC, van Leeuwen J, Attema J, et al. Detection of a bright burst from FRB 121102 with Apertif at the Westerbork Synthesis Radio Telescope. The Astronomer's Telegram. 2017; 10693.

97. Scholz P, Bogdanov S, Hessels JWT, et al. Simultaneous X-Ray, GammaRay, and Radio Observations of the Repeating Fast Radio Burst FRB 121102. ApJ. 2017; 80-90. doi: 10.3847/1538-4357/aa8456

98. Magic Collaboration, Acciari VA, Ansoldi S, Antonelli LA, et al. Constraining very-high-energy and optical emission from FRB 121102 with the MAGIC telescopes. MNRAS. 2018; 481: 2479-2486. doi: 10.1093/mnras/sty2422

99. Gajjar V, Siemion APV, Price DC, et al. Highest Frequency Detection of FRB 121102 at $4-8 \mathrm{GHz}$ Using the Breakthrough Listen Digital Backend at the Green Bank Telescope. ApJ. 2018; 863: 2. doi: 10.3847/1538-4357/aad005

100. Spitler LG, Herrmann W, Bower GC, et al. Detection of Bursts from FRB 121102 with the Effelsberg $100 \mathrm{~m}$ Radio Telescope at $5 \mathrm{GHz}$ and the Role of Scintillation. ApJ. 2018; 863: 150. doi: 10.3847/1538-4357/aad332

101. Zhang YG, Gajjar V, Foster G, et al. Fast Radio Burst 121102 Pulse Detection and Periodicity: A Machine Learning Approach. ApJ. 2018; 866(2): 149. doi: 10.3847/1538-4357/aadf31

102. Zhang BB, Zhang B. Repeating FRB 121102: Eight-year Fermi-LAT Upper Limits and Implications. ApJL. 2017; 843: L13-L17. doi: 10.3847/20418213/aa7633

103. Ofek EO. A Search for FRB 121102-like Persistent Radio-luminous Sources-Candidates and Implications for the FRB Rate and Searches. ApJ. 2017; 846: 44-53. doi: 10.3847/1538-4357/aa8310

104. Cheng B, Epstein RI, Guyer RA, Young AC. Earthquake-like behaviour of soft $\gamma$-ray repeaters. Nature. 1996; 382: 518-520. doi: 10.1038/382518a0
105. Gogus E, Woods PM, Kouveliotou C, et al. Statistical Properties of SGR 1900+14 Bursts. ApJL. 1999; 526: L93-L96. doi: 10.1086/312380

106. Gogus E, Woods PM, Kouveliotou C, et al. Statistical Properties of SGR 1806-20 Bursts. ApJL. 2000; 532. L121-L124. doi: 10.1086/312583

107. Haskell B. The effect of superfluid hydrodynamics on pulsar glitch sizes and waiting times. MNRAS. 2016. 461: L77-L81. doi: 10.1093/mnrasl/ slw103

108. Melatos A, Peralta C, Wyithe JSB. Avalanche Dynamics of Radio Pulsar Glitches. ApJ. 2008; 672: 1103-1118. doi: 10.1086/523349

109. Onuchukwu CC, Chukwude AE. A study of microglitches in Hartebeesthoek radio pulsar. Ap\&SS. 2016; 361: 300-312. doi: 10.1007/s10509-016-2835-8

110. Savchenko V, Neronov A, Beckmann V, Produit N, Walter R. SGR-like flaring activity of the anomalous $X$-ray pulsar 1E 1547.0-5408. A\&A. 2010; 510: A77-A86. doi: 10.1051/0004-6361/200911988

111. Gavriil FP, Kaspi VM, Woods PM. A Comprehensive Study of the X-Ray Bursts from the Magnetar Candidate 1E 2259+586. ApJ. 2004; 607(2): 959969. doi: $10.1086 / 383564$

112. Wang FY, Dai ZG. Self-organized criticality in X-ray flares of gamma-rayburst after glows. Nature Physics. 2013; 9: 465-467. doi: 10.1038/ nphys 2670

113. Guidorzi C, Dichiara S, Frontera F, Margutt R, Baldeschi A, Amati L. A Common Stochastic Process Rules Gamma-ray Burst Prompt Emission and X-ray Flares. ApJ. 2015; 801: 57-68. doi: 10.1088/0004-637X/801/1/57

114. Katz J. Excess close burst pairs in FRB 121102. MNRAS. 2018; 476: 18491852. doi: $10.1093 / \mathrm{mnras} / \mathrm{sty} 366$

115. Kulkarni SR, Ofek EO, Neill JD. The Arecibo Fast Radio Burst: Dense Circum-burst Medium. ApJ. 2017.

116. Price DC, Gajjar V, Rosenthal L. No Bursts Detected from FRB121102 in Two $5 \mathrm{hr}$ Observing Campaigns with the Robert C. Byrd Green Bank Telescope. Research Notes of the American Astronomical Society. 2018; 2(1): 30. doi: 10.3847/2515-5172/aaaf69

117. Niels O, Ran YH, Li PU. On the non-Poissonian repetition pattern of FRB121102. MNRAS. 2018; 475: 5109-5115. doi: 10.1093/mnras/sty004

118. Wheatland MS, Sturrock PA, McTiernan JM. The Waiting-Time Distribution of Solar Flare Hard X-Ray Bursts. ApJ. 1998; 509: 448-455. doi: $10.1086 / 306492$

119. Bagchi M. A Unified Model for Repeating and Non-repeating Fast Radio Bursts. ApJL. 2017; 838: L16-L21. doi: 10.3847/2041-8213/aa65c9

120. Katz Jl. Fast radio bursts-A brief review: some questions, fewer answers. Modern Physics Letters A. 2016; 31(14): 1630013. doi: 10.1142/ S0217732316300135 